\title{
German Pinscher
}

National Cancer Institute

\section{Source}

National Cancer Institute. German Pinscher. NCI Thesaurus. Code C54050.

The German Pinscher, also called the Standard Pinscher, is a medium-sized, tall terrier with a smooth, glossy coat that comes in red fawn, black and tan, and dark brown with yellow markings. Black with tan markings is the most common. The ears have a natural fold when they are uncropped. Cropped ears are high set. The body is well-muscled with a straight back. Height: 16-19 inches (41-48 cm.) Weight: 25-35 pounds (11-16 kg.) 